\title{
The Internet of Things (IOT) in Agriculture Monitoring
}

\author{
Alia Rifat, Prince Patel, B. Shoban Babu
}

\section{ABSTRACT}

Regardless of how the public perceives agriculture, the reality is that today's agriculture industry is more data-driven, precise, and sophisticated than ever before. The rapid rise of Internet-of-Things (IoT)based technologies has transformed practically every industry, including "smart agriculture," which has moved away from statistical to quantitative techniques. Smart agriculture allows for more efficient fertilizer use and less water waste. The goal of the smart agribusiness research is to create a dynamic and emotionally supportive farm management network. IoT is being used in agriculture to learn about the agricultural field by using sensors for field monitoring and control. Sensors are used to acquire a better understanding of the crop field for monitoring, field control, and other applications. We'll look at how Internet of Things architectures aid farm fields in this research. The entire Internet of Things (IoT) Solicitations in Agriculture, as well as how they work, are provided. According to the report, IoT can also be used to fill in gaps in agriculture. Technology scalability should be encouraged without jeopardizing existing infrastructures functionality. The current state of Internet of Things (IoT) solicitations in agricultural, as well as future projections, are discussed in this article.

\section{Keywords: Internet-of-Things (IoT), LeenaBOT, sensors, smart} agriculture.

\section{INTRODUCTION}

IoT in agriculture could be a game changer for humans and the world as a whole. Extreme weather, decreasing earth and drying parklands, as well as declining habitats that play a crucial role in agriculture, are all making food production more challenging right now. Agriculturalists will benefit from Internet of Things (IoT) technology, which will help them reduce waste and increase efficiency. It's possible that this is related to the amount of compost applied or the number of missions completed by the farmhouse vehicles [1]. As a result, smart agriculture is essentially an automated system for creating contaminant-free, crowd-pleasing sustenance. Smooth Agricultural, which uses the Internet of Things to monitor the ground in real time, improves the entire farming system. Thanks to gadgets and interconnectivity, the Internet of Things (IoT) in farming has not only saved agriculturalists' time, but it has also summarized the inefficient use of characteristics such as liquid and electricity. It remembers topographies that are regularly inspected, such as moisture, high temperature, soil, and so on, and provides crystal-clear real-time monitoring. Fig. 1 displays the primary technological drivers to wrap up this talk [2].

Some of the benefits of adopting contemporary technology in agriculture include Internet of Things in Agriculture [3], [4].
Published Online: February 15, 2022

ISSN: $2736-5492$

DOI : $10.24018 /$ ejcompute.2022.2.1.49
A. Rifat*
Hobby Research Associate, Dhaka,
Bangladesh.
(e-mail: jodhyy@gmail.com)
P. Patel
Charotar University of Science and
Technology Gujarat, India.
(e-mail: princepatel221375@gmail.com)
B. Shoban Babu
SV Engineering College Tirupati, India.
(e-mail: bshobanbabu@gmail.com)

*Corresponding Author

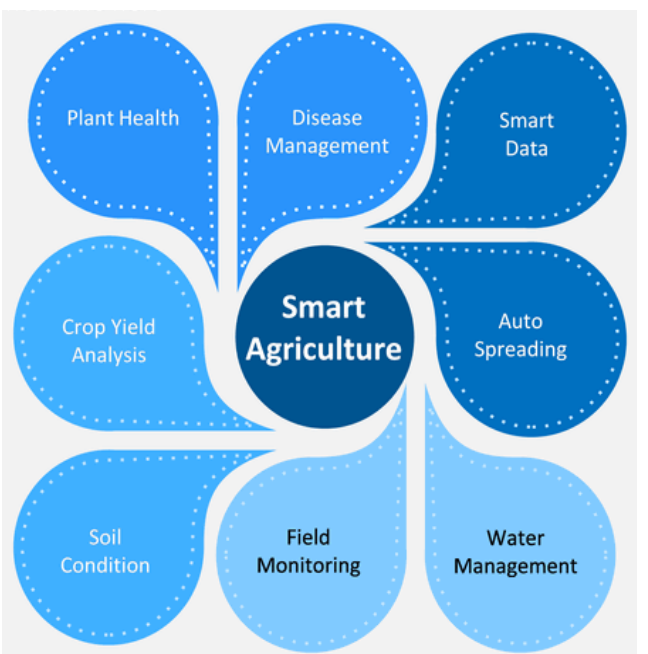

Fig. 1. Key drivers of technology in agriculture industry [5].

\section{A. Weather Conditions}

Climate is tremendously essential in agriculture. Furthermore, a lack of awareness of climate has a substantial impact on the amount and quality of agricultural products. IoT technology, on the other hand, allow you to track the weather in real time. Sensors have been put both within and outside of agriculture regions. They gather data from the environment, which is then used to determine which crops are most suited to the climate. Sensors are utilized throughout the 
Internet of Things ecosystem to track real-time weather factors like humidity, rainfall, temperature, and more.

\section{B. Precision Agriculture}

One of the most well-known IoT applications in agriculture is precision agriculture, also known as precision farming. Animal monitoring, vehicle tracking, field observation, and inventory monitoring are examples of smart farming applications that serve to make farming more precise and controlled. The goal of precision farming is to analyses data from sensors and react accordingly.

\section{Ingenious Greenhouse}

Weather stations may now automatically adjust climate conditions in response to a set of instructions thanks to the Internet of Things, allowing us to make our greenhouses smart. In greenhouses, the use of IoT has eliminated the need for human interaction, making the entire process more costeffective and accurate. For example, solar-powered IoT sensors can be utilized to build complex and low-cost greenhouses. These sensors collect and transmit real-time data, allowing for precise real-time greenhouse monitoring. Thanks to the sensors, the water usage and greenhouse condition may be tracked via emails or SMS notifications. Irrigation is carried out automatically and intelligently via the Internet of Things. Pressure, humidity, temperature, and light levels can all be measured with these sensors.

\section{Data Science}

Data from IoT devices necessitates more storage than a typical database system can give. Cloud-based data storage and an end-to-end IoT platform are critical components of the smart agriculture system. These systems are likely to play a key role in facilitating better activity execution. In the IoT world, sensors are the primary source of large-scale data collecting. The data is reviewed and turned into meaningful information using analytics technologies.

\section{E. Drones for Agriculture}

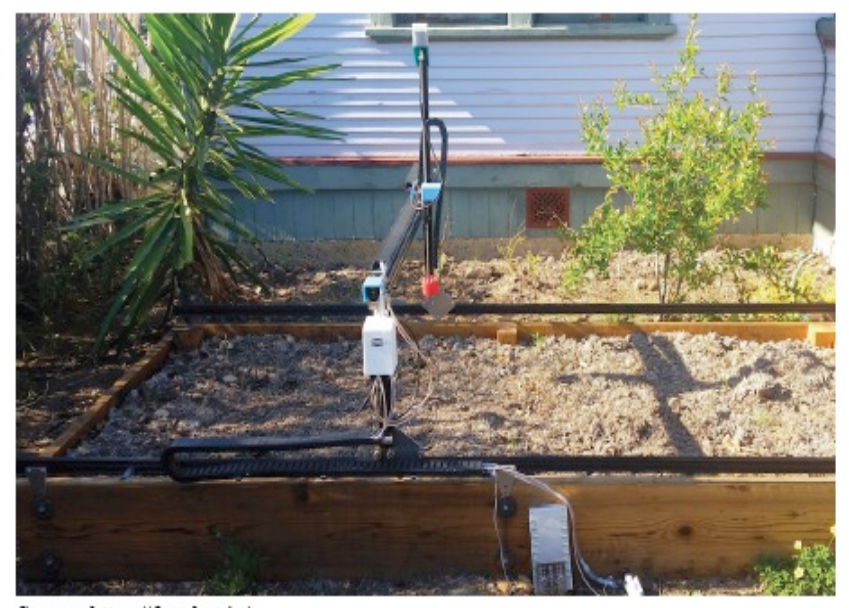

Source: https://farmbotio/

Fig. 2. FarmBot [7]

Agricultural operations have almost completely transformed as a result of technological advancements, with the most recent disruption being the introduction of agricultural drones. Drones are used for agricultural health assessments, crop monitoring, planting, crop spraying, and field analysis on the ground and in the air. Thanks to solid strategy and planning based on real-time data, drone technology has given the agriculture industry a lift and makeover. Drones with thermal or multispectral sensors detect areas where irrigation has to be adjusted. When the crops start to grow, sensors detect their health and determine their vegetation index.

One example of combining open hardware and software in IoT in agriculture is Project Farm Bot [6], which aspires to construct humanity's open-source automated precision agricultural equipment (see Fig. 2).

\section{LiterATURE REVIEW}

Reference [7] presented agricultural yields have improved significantly over the course of human history with less assets and labour efforts. Finally, during these times, the high population rate would prohibit demand and supply from joining. According to forecasts, the global population would reach 9.8 billion by 2050, roughly a quarter of a billion more than it is now.

Reference [8] proposed to raise the issue of how to feed this growing population by boosting the volume and diversity of harvest yields. This demand can be supplied by utilizing expert and astute farming cycle advancements. Farmers should also visit agribusiness sites on a regular basis over the crop's life cycle to acquire a better grasp of harvest conditions, given traditional cultivating practices. Because $70 \%$ of growing time is spent observing and understanding the state of the crop rather than performing real field work, intelligent agribusiness is required.

Reference [9] in their work in order to reduce failures and enhance outcomes across all business sectors, the Internet of Things (IoT) has just begun to impact a wide range of areas and organizations, spanning from manufacturing, health, communications, and energy to agriculture.

Reference [10] shown in the recent past, that IoT improvements to play a vital role in a range of agricultural applications. This is due to IoT's capabilities, which include the necessary communication infrastructure as well as a wide range of administrations such as local or remote data procurement, cloud-based intelligent data investigation and decision-making, client interfacing, and agribusiness activity mechanization. Such talents have the potential to alter the agriculture industry, which is currently one of our financial value chain's most inefficient components.

\section{Proposed System}

In an IoT architecture, the device layer, network layer, and application layer are all present. I automated identification (AutoID), (ii) sensing, and (iii) actuating physical objects, i.e. virtualized real-world objects, are all possible with the device layer (e.g. plant, parcel, animal, stable, box, pallet, truck). In agriculture, barcodes and RFID are two of the most used AutoID technologies. Various sensors are used to measure temperature, humidity, light wetness, $\mathrm{CO} 2$, ammonia, and $\mathrm{pH}$ levels.

Agricultural applications that use smartphone built-in sensors are largely GPS and camera-based, as demonstrated in Fig. 3. Tractor implements, climate control, irrigation, refrigerators, lights, aquaculture control systems, and food 
processing machinery are all controlled by actuators. To efficiently and securely convey object information, the network layer provides networking, connection, and transport services [11]. Finally, the application layer provides intelligence for operations involving virtual objects. It includes features such as tracking and tracing, dynamic state monitoring, event management, optimization, and autonomous objects.

In agriculture and food, the most typical IoT functionalities are tracking, tracing, monitoring, and event management. Because smart agriculture would greatly minimize modern agriculture's negative environmental externalities, we need it to spread and evolve from where it is currently. Smart cities use Internet of Things (IOT) equipment such connected sensors, lighting, and meters to collect and analyses data. It's a device that can be operated manually or automatically. This type of dual mode can help increase crop output while also guaranteeing that the gadget stays operational [12]. This form of customized design allows the equipment to work without interruption. Any of the alternate methods can be utilized immediately away to fix the device's problem.

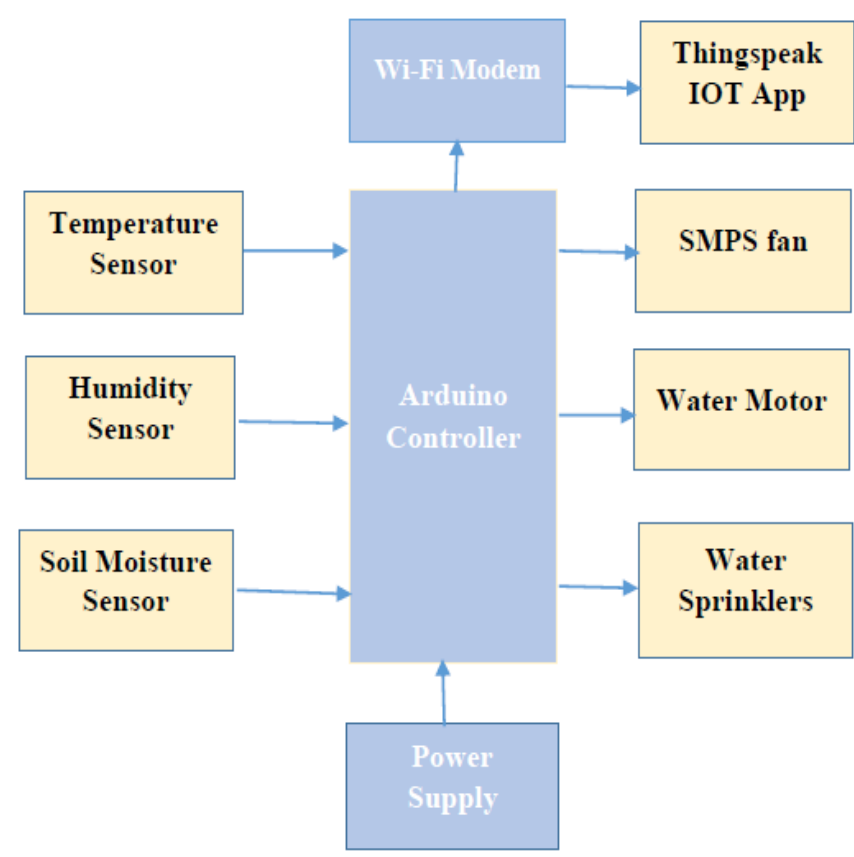

Fig. 3. Block Diagram IoT based Smart architecture.

\section{Proteus 8 Software AND Results}

Intelligent Schematic Input System application can be used to design the electric layout of the circuit, which includes resistors, coils, capacitors, a power supply, and even microprocessors. The application output is retrieved via IOT in a web server built with Thing Speak. The farmer's login id and password are unique. As illustrated in Fig 4, the data will be saved on this web server on a regular and indefinite basis. Based on changes in the environment around the field, the temperature sensor creates a graphical representation at regular intervals [13]. With the help of this graph 5, the appropriate temperature level and the insufficient temperature level may be understood.

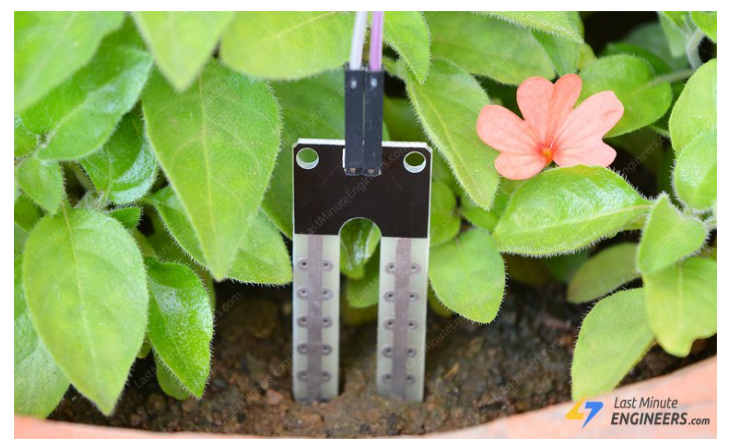

Fig. 4. Monitoring moisture sensor data using IoT.

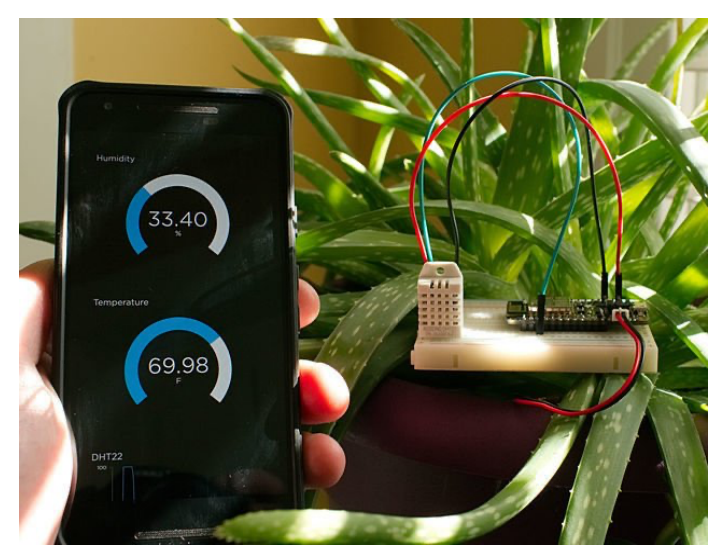

Fig.5. Monitoring temperature sensor data using IoT.

\section{Major Equipment And Technologies}

This section discusses the most important technologies and equipment currently available for this purpose.

\section{A. Wireless Sensors}

The most important and crucial sensors are wireless sensors, which are used to collect agricultural conditions and other data. Farm management chores such as soil cultivation, weeding, and fruit harvesting can all benefit from the usage of acoustic sensors. FPGA-based sensors have just recently begun to be used in agriculture due to their re-configurability. These sensors use light reflectance phenomena to assess soil organic components, soil moisture and color, mineral presence and composition, clay content, and other parameters. Ultrasonic range sensors are regarded as a great alternative due to their inexpensive cost, flexibility to perform in a number of applications, and ease of use and adjustability, such as the sample rate [14]. Common uses include tank monitoring, spray distance measurement, and crop canopy monitoring. Weeds, pesticides, and other undesired plants can be identified using optoelectronic sensors, which is especially beneficial in wide-row crops. Airflow sensors can assess soil structure and distinguish between different types of soils, as well as measure soil air permeability and moisture percentages. Electrochemical sensors are commonly used to monitor soil nutrient levels by detecting crucial soil characteristics such as $\mathrm{pH}$.

In real-world scenarios, electromagnetic sensors are used to record electrical conductivity and transient electromagnetic response, detect electrical response, and govern variable rate applications. To detect the level of compaction, mechanical sensors measure the soil's mechanical resistance. Because they detect the volume of 
grain flow and provide yield information, Mass Flow Sensors are used for yield monitoring. Using Eddy Covariance as a basis Carbon dioxide, water vapor, methane, and other gases, as well as energy exchanges between the earth's surface and the atmosphere, can all be measured with sensors. Soft Water with a Base Level Sensors are being employed in agriculture catchments to quantify hydrological phenomena including water level and flow at variable time-step acquisitions. Light detection and range are used for land mapping and segmentation, detecting soil type, farm 3D modelling, monitoring erosion and soil loss, and yield forecasting, to name a few applications. Telematics sensors provide communication between two places, or more precisely, two vehicles, in agriculture-related applications [15]. Remote sensing sensors are used to collect and store geographic data, as well as to analyze, alter, manage, and display many types of spatial and geographical data.

\section{B. Tractors Based On IOT}

Thanks to technology improvements, most of these manufacturers are now selling tractors with automatic-driven and even Cloud-computing capabilities. One of the primary advantages of self-driving tractors is their ability to avoid returning the same location or row by reducing the overlap to less than an inch. They can also make extremely precise turns without requiring the assistance of a driver. This facility allows for more precision and fewer errors, which are nearly unavoidable when the machinery is managed by a human, especially when spraying insecticide or targeting weeds.

\section{Robots for Harvesting}

In order to automate and improve the harvesting process, robots have played a larger role in recent decades in order to improve the sensitivity of fruit identification, shape, size, color, and localization. Many robots are being created for certain crops because of their intricacy. SW 6010 and Octinion for strawberries, Sweeper robot for peppers, and FFRobot for tree-based fruits like apples, which can take up to 10,000 fruits per hour, are some of the most popular crop harvesting robots.

\section{Agricultural Communication}

Communication and timely information reporting are the backbones of precision agriculture. In the agriculture industry, telecom operators can play a vital role in ensuring communication reliability. Low-power wide-area network (LPWAN) networks are appropriate, and they can be utilised in a range of other farming-related applications due to their effectiveness. Mesh networks enable peer-to-peer wireless connections by utilizing a number of communication technologies such as Bluetooth and Zigbee. In applications like as irrigation and fertilization, Zigbee modules are networked for communication. For example, drip irrigation is used to monitor soil contents such as moisture. In addition, the farmer receives SMS updates on field data, where GSM is required for long distances and a Bluetooth module can aid for shorter distances. Bluetooth has evolved in many IoT systems since the introduction of Bluetooth Low Energy (BLE), often known as Bluetooth Smart. Bluetooth is a wireless communication technology that connects small-head devices over short distances in order to allow them to communicate with one another [16]. The deployed WSN nodes communicate wirelessly with a central server, which collects and stores the monitored data as well as allows the information to be displayed after necessary analysis. LoRa is a low-power, long-range wireless technology commonly used in Internet of Things (IoT) applications. Sigfox is also used to provide network access to low-power devices that are required as "things." By collecting very small amounts of spectrum and modifying the phase of the carrier radio wave, it uses narrowband or ultra-narrowband technology to encode data [17].

\section{E. Smartphones}

When the need to contact or update the majority of the farming community arises, cell phones are a widely used and primary mode of communication. A farmer's curiosity may be piqued by a simple-to-use, free or low-cost app that supports various languages.

\section{F. Computing in the Cloud}

Farmers benefit from cloud computing because they can access knowledge-based repositories that contain a plethora of information and experiences regarding farming operations, as well as equipment possibilities on the market with all of the necessary details.

\section{HARDWARE IMPLEMENTATION}

It's a device that can be operated manually or automatically. This type of dual mode can help increase crop output while also guaranteeing that the gadget stays operational. This form of customized design allows the equipment to work without interruption. Any of the alternate methods can be utilized immediately away to fix the device's problem. Fig. 6 shows the whole agricultural automation process in operation, as depicted by the hardware prototype.

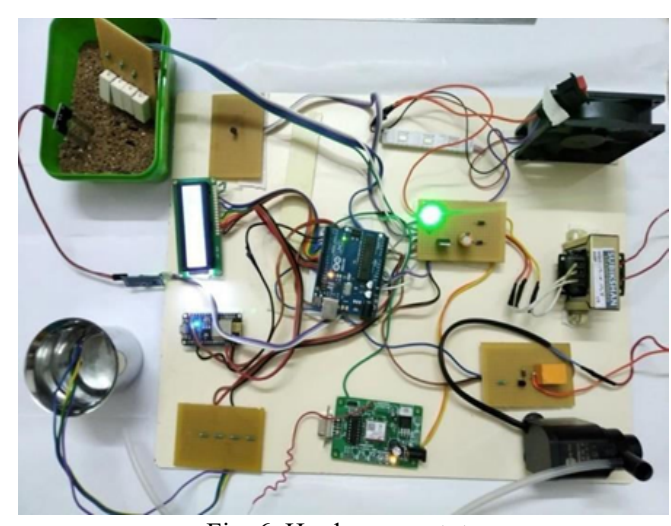

Fig. 6. Hardware prototype.

With the use of button 1, the LCD will automatically display the mode in which the device is operating while it is working. So that the entire process may be automated, and the proper amount of water and nutrients can be monitored and provided to the soil.

\section{CONCLUSION}

The Internet of Things (IoT) is becoming increasingly important, and it is rapidly evolving in a variety of fields. As a result of this expansion, various new technological 
advancements as well as new issues have evolved. A vast number of IoT devices in use or in development must be categorized according to their purpose, type, internet connectivity, deployment location, and other characteristics. Agriculture powered by the Internet of Things has aided in the deployment of cutting-edge technical solutions to age-old knowledge. This has helped to close the gap in output, quality, and yield. Quick action and minimal crop damage are ensured by data acquired by acquiring and importing data from multiple sensors for real-time use or storage in a database. Due to end-to-end intelligent operations and increased business process execution, produce is processed faster and reaches supermarkets in the shortest time feasible. This would be tremendously beneficial in terms of water management, crop selection, and chemical fertilizer and pesticide consumption reduction. It also aids farmers in their efforts to improve their financial status. It is possible to cultivate the development of a healthy crop.

\section{REFERENCES}

[1] R.Satheesh Kumar, D. Kanimozhi, S. Saravanan. An Efficient Control Scheme for Wind Farm Using Back to Back Converter. International Journal of Engineering Research \& Technology. 2013; 2(9): 32823289.

[2] Prakashraj K, Vijayakumar G, Saravanan S, Saranraj S. IoT Based Energy Monitoring and Management System for Smart Home Using Renewable Energy Resources. International Research Journal of Engineering and Technology. 2020; 7(2): 1790-1797.

[3] Mohammed Siddi J, Senthil Kumar A, Saravanan S, Swathisriranjani M. Hybrid Renewable Energy Sources for Power Quality Improvement with Intelligent Controller. International Research Journal of Engineering and Technology. 2020; 7(2): 1782-1789.

[4] Kanade P, Ashwini P. Smart Agriculture Robot for Sowing Seed. International Journal of Engineering Science and Computing. 2021; 11(1): 27563-27565.

[5] Raveendar S, Manikandan PM, Saravanan S, Dhinesh V, Swathisriranjani M. Flyback Converter Based BLDC Motor Drives for Power Device Applications. International Research Journal of Engineering and Technology. 2020; 7(2): 1632-1637.

[6] Kanade P, Prasad JP. Arduino based Machine Learning and IoT Smart Irrigation System. International Journal of Soft Computing and Engineering. 2021; 10(4): 1-5.

[7] Ayaz M, Ammad-Uddin M, Sharif Z, Mansour A, Aggoune E,-H.M. Internet-of-Things (IoT)-Based Smart Agriculture: Toward Making the Fields Talk. IEEE Access. 2019; 7, 129551-129583.

[8] Navulur S, Sastry MN, Prasad G. Agricultural Management through Wireless Sensors and Internet of Things International Journal of Electrical and Computer Engineering. 2017; 7(6):3492-3499.

[9] Sisinni E, Saifullah A, Han S, Jennehag U, Gidlund M. Industrial Internet of Things: Challenges, Opportunities, and Directions. IEEE Transactions on Industrial Informatics. 2018; 14(11): 4724-4734.

[10] Elijah O, Rahman TA, Orikumhi I, Leow CY, Hindia MN. An Overview of Internet of Things (IoT) and Data Analytics in Agriculture: Benefits and Challenges. IEEE Internet of Things Journal. 2018; 5(5): 3758-3773.

[11] Vignesh TR, Swathisriranjani M, Sundar R, Saravanan S, Thenmozhi T. Controller for Charging Electric Vehicles Using Solar Energy. Journal of Engineering Research and Application. 2020; 10(1): 49-53.

[12] Kanade P, Prasad JP. Machine Learning Techniques in Plant Conditions Classification and Observation. IEEE 2021 5th International Conference on Computing Methodologies and Communication (ICCMC). 2021, 729-734.

[13] Al-Fuqaha A, Guizani M, Mohammadi M, Aledhari M, Ayyash M. Internet of Things: A Survey on Enabling Technologies, Protocols, and Applications. IEEE Communications Surveys \& Tutorials. 2015; 17(4): 2347-2376.

[14] Kanade P, Alva P, Kanade S, Ghatwal S. Automated Robot ARM using Ultrasonic Sensor in Assembly Line. International Research Journal of Engineering and Technology (IRJET). 2020; 7(12): 615-620.

[15] Atzori L, Iera A, Morabito G. The Internet of Things: A survey. Computer Networks. 2010; 54(15): 2787-2805.
[16] Kanade P, Alva P, Prasad JP, Kanade S. Smart Garbage Monitoring System using Internet of Things(IoT). IEEE 2021 5th International Conference on Computing Methodologies and Communication (ICCMC). 2021: 330-335.

[17] Ayaz M, Ammaduddin M. Internet-of-Things (IoT)-Based Smart Agriculture: Toward Making the Fields Talk. IEEE Access. 2019; 7. 